\title{
ROUGH SETS REDUCTION FOR BINARY DATA
}

\author{
S.M.Attawey*, A.N.Abady*, A.M.Kozae ${ }^{* *}$, E.A.Rady*** \\ * Department of Engineering Mathmatics \& Basic Science, Faculty of \\ Engineering, Minoufiya University, Shebin El-Kom, Egypt \\ ** Department of Engineering Mathmatics \& Basic Science, Faculty of \\ Engineering, Tanta University, Egypt \\ *** Institute of Statistical Studies \& Research (ISSR), Cairo University, Egypt.
}

\begin{abstract}
The major aim of this work is to introduce a new method for data reduction in information systems classified by nonequivalence relations. The suggested approach is useful for many real life data that can not be classified into disjoint classes. The new approach is tested with examples and its ability for decreasing the noisy in data, as well as simplifying the structure for extracting decision rules. Based on binary relation, this method is capable of discovering the reduct attributes or indispensable attributes which are useful for data description and/or prediction, and to remove the dispensable ones.

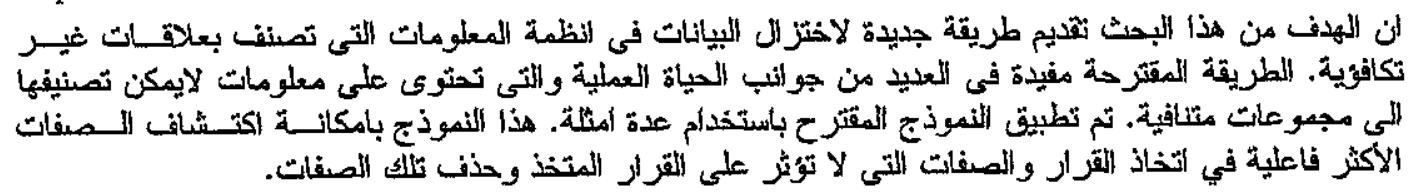

Keywords: Reduction, information system, binary relation

\section{INTRODUCTION}

Rough set theory is a mathematical approach for handling vagueness and uncertainty in data analysis. It was first introduced by Zidilaw Pawlak in the 1982 [7]. The idea of the rough set proves to be very useful in practice where many real life applications have been implemented such as civil engineering [1], medical data analysis [9], synthesis of switching circuit [5] among other. Many rough sets models have been developed in the rough set community in the last decades $[3,7,10,11,12,13,14]$ including ziarako's Variable Precision Rough Set Model (VPRS) [14] and HU's Generalized Rough Sets Model (GRS) [3], to name a few. These rough sets models focused on the extension of the original model proposed by Pawlak $[7,8]$ and attempted to deal with its limitations such as handling statistical distribution or noisy data.

In this paper, we overcome the problem of information systems which cannot classified using equivalence relation.

The rest of the paper is organized as follows: We give an overview of the classical rough set theory based on the model proposed by Pawlak $[7,8]$ with some example in section 2 and 3 . In section 4 , we redefine the main concepts and methods of rough set theory based. on binary information data, and illustrate these concepts with examples. With the new definition, we propose a new method to compute the core and reduct. Finally, we conclude with some discussions and future works in section 5.

\section{PRELIMINARIES}

In rough sets theory the data collected in a table called decision table. Rows of decision table correspond to objects, and columns correspond to attributes. In the data set, it is assumed that we are given a set of examples with a class label to indicate the class to which each example belongs. We call the class label the decision attribute, the rest of attributes, the condition attributes. Rough set theory defines three regions based on the equivalence classes induced by the attributes values; lower approximation, upper approximation and boundary region. Further more rough sets theory classifies all the attributes into three categories : core attributes, reduct attributes and dispensable attributes.

We give the formal definition as below:

\section{Definition 2.1}

Let $I=(U, A)$ be an information system, where $U$ is a non-empty set of finite objects (the universe of discourse); $A$ is a non-empty finite set of attributes such that $a: U \rightarrow V_{a} \forall \mathrm{a} \in \mathrm{A}, \mathrm{V}_{\mathrm{a}}$ being the value set of attribute $a$. in a decision system, $A=\{C \cup D\}$ where $C$ is the set of conditional attributes and $D$ is the set of decision attributes. With any $\mathrm{B} \subseteq \mathrm{A}$ there is an associated equivalence relation $\mathrm{IND}(\mathrm{B})$ : 


$$
I N D(B)=\{(x, y) \hat{I} U \times U \mid " a \hat{I} B, a(x)=a(y)\}
$$

\section{Definition 2.2}

Let $\mathrm{U}$ be a universe and $\mathrm{X} \subseteq \mathrm{U},[x]_{B}$ denotes the equivalence classes of the $\mathrm{B}$-indiscernibility relation then the $\mathrm{B}$ - lower approximation $\underline{B} X$ under $B \subseteq A$ is defined as:

$\underline{B} X=\cup\left\{x \in U \mid[x]_{B} \subseteq X\right\}$

\section{Definition 2.3}

Let $U$ be a universe and $\mathrm{X} \subseteq \mathrm{U}$ the $\mathrm{B}$ - upper approximation $\underline{B} X$ under $B \subseteq A$ is defined as :

$\bar{B} X=\cup\left\{x \in U \mid[x]_{B} \cap X \neq \phi\right\}$

\section{Definition 2.4}

Let $B$ and $Q$ be equivalence relations over $U$ then the positive, negative and boundary reign can be defined as:

$$
\begin{aligned}
& \operatorname{POSB}(Q)=\bigcup_{x \in U / Q} B(X) \\
& N E G B(Q)=U-\bigcup_{x \in U / Q} \overline{B X} \\
& B N D B(Q)=\bigcup_{x \in U / Q} \bar{B} X-\bigcup_{x \in U / Q} B X
\end{aligned}
$$

In terms of classification, the positive region contains all objects of $U$ that can be classified to classes of $U / Q$ using knowledge in attributes $B$.

\section{Definition 2.5}

For $\mathrm{P}, \mathrm{Q} \subseteq \mathrm{A}, \mathrm{Q}$ depend on $\mathrm{P}$ in a degree $K(0 \leq K \leq 1)$, denoted $\mathrm{P} \Rightarrow{ }_{k} \mathrm{Q}$

$$
K=\gamma_{P}(Q)=\frac{\operatorname{card}(\operatorname{POS} P(Q))}{\operatorname{cord}(U)}
$$

If $K=1, Q$ depend totally on $P$.

$$
\text { If } 0 \prec K \prec 1 \text {, } \mathrm{Q} \text { depend partially on } \mathrm{P} \text {. }
$$

If $K=0, Q$ does not depend on $P$.

To illustrate this definition we give the following example;

\section{Example 2.1}

$$
\text { Let } U=\{0,1,2,3,4,5,6,7\}
$$

$$
\begin{aligned}
& U / P=\{\{2\},\{0,4\},\{3\},\{1,6,7\},\{5\}\} \\
& U / Q=\{\{0\},\{1,3,6\},\{2,4,5,7\}\} \\
& \operatorname{Pos} p(Q)=\{2,3,5\} \\
& K=\gamma_{p}(Q)=\frac{|\{2,3,5\}|}{|\{0,1,2,3,4,5,6,7\}|}=\frac{3}{8}
\end{aligned}
$$

\section{Definition 2.6}

By calculating the change in dependency when an attribute is removed from the set of considered conditional attributes, a measure of significance of attribute (coefficient of significance) more formally,

If $\mathrm{P}, \mathrm{Q} \subseteq \mathrm{A}$ and an attribute $\mathrm{x} \in \mathrm{P}$ the significance of attribute $\mathrm{x}$ upon $\mathrm{Q}$ is defined by

$$
\partial_{P}(Q, x)=\gamma_{P}(Q)-\gamma_{P-\{x\}}(Q)
$$

If the significance is zero then the attribute is dispensable, the bigher $\partial$ the more significance attribute.

To identify the above definition:

$$
\begin{gathered}
\text { Let } U=\{0,1,2,3,4,5,6,7\} \\
U / a=\{\{0,3,4\},\{1,7\},\{2,5,6\}\} \quad U / b=\{\{0,2,4\},\{1,3,6,7\},\{5\}\} \\
U / c=\{\{0,4\},\{1,6,7\},\{2,3,5\}\} \quad U / e=\{\{0\},\{1,3,6\},\{2,4,5,7\}\} \\
P=\{a, b, c\} \quad Q=\{e\} \\
U / P=\{\{0,4\},\{1,7\},\{2\},\{3\},\{5\},\{6\}\} \\
U / Q=\{\{0\},\{1,3,6\},\{2,4,5,7\}\} \\
U / P-\{a\}=\{\{2\},\{0,4\},\{3\},\{1,6,7\},\{5\}\} \\
U / P-\{b\}=\{\{0,4\},\{1,7\},\{3\},\{2\},\{6\},\{5\}\} \\
U / P-\{c\}=\{\{0,4\},\{2\},\{3\},\{1,7\},\{6\},\{5\}\} \\
P o s_{p}(Q)=\{2,3,5,6\} \quad P a s_{p-(a)}(Q)=\{2,3,5\} \\
P o s_{p-\{b\}}(Q)=\{2,3,5,6\} \quad P_{p-(c)}(Q)=\{2,3,5,6\} \\
\gamma_{p}(Q)=\frac{4}{8} \quad \gamma_{p \sim(a)}(Q)=\frac{3}{8} \\
\gamma_{p-(b)}(Q)=\frac{4}{8} \quad \gamma_{p-\{a)}(Q)=\frac{4}{8}
\end{gathered}
$$

by calculating the signifcance of the three attributes gives:

$$
\begin{aligned}
& \partial_{P}(Q, a)=\gamma_{P}(Q)-\gamma_{P-\{a\}}(Q)=\frac{1}{8} \\
& \partial_{P}(Q, b)=\gamma_{P}(Q)-\gamma_{P-\{b\}}(Q)=0 \\
& \partial_{P}(Q, c)=\gamma_{P}(Q)-\gamma_{P-\{c\}}(Q)=0
\end{aligned}
$$

\section{Reduct and core}

Some attributes of the information system may be redundant with respect to a specific classification. Attributes are removed so that the reduced set 
provides the same quality of classification as the original. In the context of decision systems, a reduct is formally defined as

\section{Definitio 2.7}

A subset $C^{\prime}$ of the attribute set $C$ is a reduct of $C$ with respect to $D$, if and only if the following conditions are satisfied:-

$$
\begin{aligned}
& { }^{1-} \operatorname{POS}_{C^{\prime}}^{(D)=} P O S_{C^{(D)}} \\
& 2-\forall R \subset C^{\prime} \Rightarrow P O S_{R}(D) \neq P O S_{C}(D)
\end{aligned}
$$

The intersection of all reduct sets is called the core , the elements of which are those attributes that cannot be eliminated without introducing contradiction to the data set.

In other words, core attributes have the essential information to make correct classification for the data set. A core is formally defined as:-

\section{Definition 2.8}

An attribute $C_{j} \in C$ is a core attribute in $\mathrm{C}$ with respect to $D$ if

$$
P O S_{c}(D) \neq P O S_{C-C}(D)
$$

Now we can define the dispensable attributes which are those redundant attributes in the data set and should be eliminated, a dispensable attribute is formally defined as

\section{Definition 2.9}

An attribute $C_{j} \in C$ is a dispensable attribute in $\mathrm{C}$ with respect to $D$ if

$$
P O S_{c}^{(D)}=\operatorname{POS}_{C-C_{j}}^{(D)}
$$

\section{A NEW PROPOSED ROUGH SETS MODEL}

There exist some limitations of rough sets theory which restrict its suitability in practice $[3,4,10,11,12,13,14]$, two of which are described as follows:

(1) Rough sets theory uses the strict set inclusion definition to define the lower approximation, which does not consider the statistical distribution noise of the data in the equivalence class. This drawback of the original rough set model has limited its applications in domains where tends to be noisy or dirty. So, some new models have been proposed to overcome this problem such as Ziarko's Variable Precision Rough Set Model (VPRS) [14].

(2) Another drawback of rough sets theory is the inefficiency in computation, which limits its suitability for large data sets in real-world applications. In order to find the reducts, core and dispensable attributes, the rough sets model need to construct all the equivalence classes based on the attribute values of the condition and decision attributes. This process is very time-consuming, and thus the model is very inefficient and infeasible, and does not scale for large data set, which is very common in data mining applications $[3,4]$. Some new algorithms to overcoming this inefficiency have been developed $[2,6]$.

Our research deal with the limitation of intersected classes and introduce a new approach to find the core and reducts based on binary data. To fit with the new approach we need some new definitions. It can be considered as a modifications for the existing definition to obtain our new approach.

\section{Right image of an element}

Let $U$ be a universe, $R$ a binary relation over $U$ and $\mathrm{x} \in \mathrm{U}$ then the Right image of an element defined as;

$$
x R=\{y: x R y\}
$$

\section{Right sub base of $\mathbf{R}$}

Let $U$ be a universe, $R$ a binary relation over $U$ and $\mathrm{x} \in U$ then the Right sub base of $\mathrm{R}$ defined as:

$$
S=\{x R: x \in U\}
$$

\section{Right base}

Let $U$ be a universe, $R$ a binary relation over $U$ and $n$ be the number of elements of $S$ the right base $(B)$ is the finite intersection of the elements of S, formally defined as:

$$
\begin{aligned}
& B=\left\{\bigcap_{i=1}^{n} B_{i}\right\} \cup\left\{\bigcap_{i=2}^{n} B_{i}\right\} \cup \ldots \ldots \ldots \ldots\left\{\bigcap_{i=n}^{n} B_{i}\right\} \\
& B=\left\{\bigcap_{i=1}^{n} B_{i}: \mathrm{B}_{\mathrm{i}} \in S, i \in\{1,2, \ldots \ldots \ldots \ldots . . . n\}\right\}
\end{aligned}
$$

\section{Right minimal base}

Let $U$ be a universe, $R$ a binary relation over $U$ and $\mathrm{B}$ the right base . then the right minimal base $\left(B_{m}\right)$ is the $\mathrm{B}$ difference the family of unions of members of element in $\mathrm{B}$ formally defined as:

$$
\mathrm{B}_{\mathrm{m}}=\mathrm{B}-\left\{\mathrm{F} \in \mathrm{B}: \mathrm{F}=\mathrm{UB}_{\mathrm{i}}, B_{i} \in B\right\}
$$

\section{Indicernibility relation}

Let $U$ be a universe, $R$ a binary relation over $U$ and $\mathrm{B} \subseteq \mathrm{R}$ then $\mathrm{IND}(\mathrm{B})$ defined as follow:

1- find $B_{R t} \quad \forall R \in B$

2- define $S=\bigcup_{R \in B} B_{R m}$

3- find Sbase

4- find $\mathrm{S}_{\min }$ base $=\mathbb{N N D}(\mathrm{B})$ 
In order to show that we give the following example,

$$
\begin{aligned}
& B m=\{\{a, b, c\},\{d, e, f\},\{b, c\},\{d, E\},\{c\},\{d\}\} \\
& X=\{a, b, d\} \\
& \underline{B}(X)=\{d\} \\
& \underline{B}(\underline{B}(X))=\{d\} \\
& U-\underline{B}(X)=\{a, b, c, e, f\} \\
& (U-\underline{B}(X))=\{a, b, c\} \\
& \bar{B}(\underline{B}(X))=\{d, e, f\}
\end{aligned}
$$

\section{Approximation of sets Via the new approach}

Let $\mathrm{G}=(\mathrm{U}, \mathrm{R})$ be binary relation system, $\mathrm{B} \subseteq R$ and $\mathrm{X} \subseteq U$. we can approximate $\mathrm{X}$ using only the information contained in $\mathrm{B}$ by constructing the $\mathrm{B}-$ Lower and B-Upper approximation defined as following:

(i) B-Lower approximation of $\mathbf{X}$

Let $\mathrm{U}$ be a universe, $\mathrm{R}$ a binary relation system over $\mathrm{U}, \mathrm{B} \subseteq R, B_{m}$ the right minimal base of $\mathrm{B}$, $X \subseteq B_{m}$ the lower approximation of $\mathrm{X}$ is defined as:

$$
\underline{B} X=\bigcup\{b: \mathrm{b} \subseteq \mathrm{X}, \mathrm{b} \in \mathrm{B}\}
$$

(ii) B-Upper approximation of $X$

Let $U$ be a universe, $\mathrm{R}$ a binary relation system over $\mathrm{U}, \mathrm{B} \subseteq R, B_{m}$ the right minimal base of $\mathrm{B}$, $X \subseteq B_{m}$ the upper approximation defined as:

$$
\bar{B} X=\{U-(U-X)\}
$$

To show that

$$
\begin{aligned}
& \text { Let } U=\{a, b, c, d, e, f\} \\
& B_{m}=\{\{a, b, c\},\{d, e, f\},\{b, c\},\{d, e\},\{c\},\{d\}\} \\
& X=\{a, b, d\} \\
& \underline{B} X=\{d\} \\
& U-X=\{c, e, f\} \\
& \underline{U}-X=\{c\} \\
& \bar{B} X=\{a, b, d, e, f\}
\end{aligned}
$$

It is easy to see the lower approximation is the same as those in the originalrough sets $[7,8]$, but the upper approximation is different from them. We can conclude the following remark.

\section{Remark}

It is easy to see the difference between the suggested approach and the old one via the following example.

Consider $Y=\{b, c, d\}$ following the above definitions the following results can be given:
$\underline{B} \mathrm{Y}=\{\mathrm{b}, \mathrm{c}, \mathrm{d}\}$

$\mathrm{U}-\mathrm{Y}=\{\mathrm{a}, \mathrm{d}, \mathrm{e}, \mathrm{f}\}$

$\underline{\mathrm{U}-\mathrm{Y}}=\{d, e, f\}$

$\bar{B} Y=\{a, b, c\}$

Notice that $\bar{B} Y \neq B Y$ and this illustrate the difference between the two approaches. In the old approach they have to be equal.

Properties of the new approximation :-

Directly from the definitions of approximations we can get the following properties of the R-Lower and R-Upper approximations:-

(Li) $\underline{R}(U)=U$ (4L) $\underline{R}(X \cap Y)=\underline{R}(X) \cap \vec{R}(Y)$

(1U) $\bar{R}(U)=U(4 \mathrm{U}) \overline{\mathrm{R}}(\mathrm{X} \cup \mathrm{Y})=\bar{R}(X) \cup \bar{R}(Y)$

(2L) $\underline{R}(\phi)=\phi \quad(5 L) \quad \underline{R}(\underline{R}(X))=\underline{R} X$

(2U) $\bar{R}(\phi)=\phi \quad(5 U) \bar{R}(\bar{R}(X))=\bar{R}(X)$

(3L) $\underline{\mathrm{R}}(\mathrm{X}) \subseteq \mathrm{X}(6 L) \underline{R}(-X)=-\bar{R}(X)$

(3U) $X \subseteq \bar{R}(X)(6 U) \bar{R}(-X)=-\underline{R}(X)$

$(7 L) \mathrm{X} \subseteq \mathrm{Y} \Rightarrow \underline{\mathrm{R}}(\mathrm{X}) \subseteq \underline{\mathrm{R}}(\mathrm{Y})(8 \mathrm{U}) \overline{\mathrm{R}}(-\overline{\mathrm{R}}(\mathrm{X}))=\overline{\mathrm{R}}(\mathrm{X})$

(7U) $\mathrm{X} \subseteq \mathrm{Y} \Rightarrow \overline{\mathrm{R}}(\mathrm{X}) \subseteq \overline{\mathrm{R}}(\mathrm{Y})(\mathrm{L}) \forall \mathrm{K} \in \mathrm{U} / \mathrm{R}, \underline{\mathrm{R}}(\mathrm{K})=\mathrm{K}$

(8L) $\underline{R}(-\underline{R}(X))=\underline{R}(X) \quad$ (9U) $\forall K \in U / R, \bar{R}(K)=K$

\section{Proposition1}

The following properties don't hold generally.
(1L) $\underline{\mathrm{R}}(\underline{\mathrm{R}}(\mathrm{X}))=\overline{\mathrm{R}}(\underline{\mathrm{R}}(\mathrm{X}))$
(1U) $\bar{R}(\bar{R}(X))=\underline{R}(\bar{R}(X))$

This can be verified via the following example;

\section{Example 3.2}

Let $\mathrm{U}=\{\mathrm{a}, \mathrm{b}, \mathrm{c}, \mathrm{d}, \mathrm{e}, \mathrm{f}\}$

$B m=\{\{a, b, c\},\{d, e, f\},\{b, c\},\{d, E\},\{c\},\{d\}\}$

$X=\{a, b, d\}$

$\underline{B}(X)=\{d\}$

$\underline{B}(\underline{B}(X))=\{d\}$

$U-\underline{B}(X)=\{a, b, c, e, f\}$

$(U-\underline{B}(X))=\{a, b, c\}$

$\bar{B}(\underline{B}(X))=\{d, e, f\}$

For the same example we note that

$\bar{B}(X)=\{a, b, d, e, f\}$

$\underline{B}(\bar{B}(X))=\{d, e, f\}$

$B-\bar{B}(X)=\{c\}$

$\underline{B}(B-\vec{B}(X))=\{c\}$

$\bar{B}(\bar{B}(X))=\{a, b, d, e, f\}$ 


\section{Degree of dependency of knowledge}

Let $G=(U, R)$ binary relation system $B_{m P}, B_{m \ell} \subseteq R$ we say the knowledge $B_{m Q}$ depends in a degree $\mathrm{E}(0 \leq E \leq 1)$ from knowledge $B_{m P}$, symbolically $B_{m P} \Rightarrow_{E} B_{m Q}$ if and only if

$E=\lambda_{B_{m P}}\left(B_{m Q}\right)=\frac{\operatorname{Card} \operatorname{POS}_{\mathrm{B}_{\mathrm{mP}}}\left(B_{m Q}\right)}{\operatorname{Card} \mathrm{U}}$

- If $\mathrm{E}=1 \Rightarrow B_{m Q}$ is totally depends from $B_{m P}$

- If $0<\mathrm{E}<1 \Rightarrow B_{m Q}$ is partially depends from $B_{m P}$

- If $\mathrm{E}=0 B_{m Q}$ in not totally depends from $B_{m P}$

The following example show the above concept

Let $U=\{a, b, c, d, e, f\}$

$B_{m P}=\{\{a, b, d, e\},\{b, c, e\},\{b, e, f\}\}$

$B_{m Q}=\{\{a, b, e ; d, f\},\{b, c\},\{d, e, f, b\}\}$

One can get the degree of dependency as follows, $P O S_{B_{m p}}\left(B_{m Q}\right)=\{a, b, d, e, f\}$

$\Rightarrow E=\lambda_{B_{m P}}\left(B_{m Q}\right)=\frac{\operatorname{Card~} P O S_{B_{m p}}\left(B_{m Q}\right)}{\operatorname{Card} \mathrm{U}}=\frac{5}{6}$

\section{Data Reduction in Binary Data}

This section is devoted to introduce the new approach for data reduction in binary data. The suggested new method is based on non-equivalence relation. So many definition will be given according to that as following.

\section{Reduct and Core}

Let $\mathrm{G}=(\mathrm{U}, \mathrm{R})$ binary information system, a subset $C^{\prime}$ of the attribute set $C$ is a reduct of $C$ with respect to $D$, if and only if the following conditions are satisfied:-

1- $P O S_{c_{m}^{\prime}}\left(D_{m}\right)=P O S_{c_{m t}}\left(D_{m}\right)$

$2-\forall R \subset C^{\prime} \Rightarrow P O S_{R_{m}}\left(D_{m}\right) \neq P O S_{C_{m}}\left(D_{m}\right)$

The intersection of all reduct sets is called the core , the elements of which are those attributes that cannot be eliminated without introducing contradiction to the data set.

In other words, core attributes have the essential information to make correct classification for the data set.
$B m=\{\{a, b, c\},\{d, e, f\},\{b, c\},\{d, E\},\{c\},\{d\}\}$

$X=\{a, b, d\}$

$\underline{B}(X)=\{d\}$

$\underline{B}(\underline{B}(X))=\{d\}$

$U-\underline{B}(X)=\{a, b, c, e, f\}$

$(U-\underline{B}(X))=\{a, b, c\}$

$\bar{B}(\underline{B}(X))=\{d, e, f\}$

For the same example we note that

$\bar{B}(X)=\{a, b, d, e, f\}$

$\underline{B}(\bar{B}(X))=\{d, e, f\}$

$B-\bar{B}(X)=\{c\}$

$\underline{B}(B-\vec{B}(X))=\{c\}$

$\bar{B}(\bar{B}(X))=\{a, b, d, e, f\}$

Degree of dependency of knowledge

Let $G=(U, R)$ binary relation system $B_{m P}, B_{m Q} \subseteq R$ we say the knowledge $B_{m Q}$ depends in a degree $\mathrm{E}(0 \leq E \leq 1)$ from knowledge $\quad B_{m P}$, symbolically $B_{m P} \Rightarrow_{E} B_{m Q}$ if and only if

$E=\lambda_{B_{m p}}\left(B_{m Q}\right)=\frac{\operatorname{Card} \operatorname{POS}_{\mathrm{B}_{m^{p}}}\left(B_{m Q}\right)}{\operatorname{Card} \mathrm{U}}$

- If $\mathrm{E}=1 \Rightarrow B_{m Q}$ is totally depends from $B_{m p}$

- If $0<\mathrm{E}<1 \Rightarrow B_{m Q}$ is partially depends from $B_{m P}$

- If $\mathrm{E}=0 \quad B_{m Q}$ in not totally depends from $B_{m P}$

The following example show the above concept

Let $U=\{a, b, c, d, e, f\}$

$B_{m P}=\{\{a, b, d, e\},\{b, c, e\},\{b, e, f\}\}$

$B_{m Q}=\{\{a, b, e, d, f\},\{b, c\},\{d, e, f, b\}\}$

One can get the degree of dependency as follows,

$P O S_{B_{m u^{p}}}\left(B_{m Q}\right)=\{a, b, d, e, f\}$

$\Rightarrow E=\lambda_{B_{m P}}\left(B_{m Q}\right)=\frac{\operatorname{Card} P O S_{B_{m P}}\left(B_{m Q}\right)}{\operatorname{Card} \mathrm{U}}=\frac{5}{6}$

Data Reduction in Binary Data

This section is devoted to introduce the new approach for data reduction in binary data. The suggested new method is based on non-equivalence 
relation. So many definition will be given according to that as following.

\section{Reduct and Core}

Let $\mathrm{G}=(\mathrm{U}, \mathrm{R})$ binary information system, a subset $C^{\prime}$ of the attribute set $\mathrm{C}$ is a reduct of $\mathrm{C}$ with respect to $D$, if and only if the following conditions are satisfied:-

$$
\begin{aligned}
& 1-P O S_{C_{m}^{\prime}}\left(D_{m}\right)=P O S_{C_{m}}\left(D_{m}\right) \\
& 2-\forall R \subset C^{\prime} \Rightarrow P O S_{R m}\left(D_{m}\right) \neq P O S_{C_{m}}\left(D_{m}\right)
\end{aligned}
$$

The intersection of all reduct sets is called the core, the elements of which are those attributes that cannot be eliminated without introducing contradiction to the data set.

In other words, core attributes have the essential information to make correct classification for the data set.

\section{Example 3.3}

Consider the data as shown in table (1) in question concern modeling of the energy for unfolding of a protein(tryptophane synthas alpha unit of the bacteriophage T4 lysozome., where 7 coded amino acids (AAs). The (AAs) are described in terms of three attributes : $a 1=$ PIE and $a 2=$ PIF (two measures of the side chain lipophilicity., a3 = DGR= $\Delta G$ of transfer from the protein interior to water and $\mathrm{d}$ is the decision attribute.

Table (1) Original information system; condition attributes $\{\mathrm{a} 1, \mathrm{a} 2, \mathrm{a} 3\}$ decision attribute $\{\mathrm{d}\}$.

\begin{tabular}{|c|c|c|c|c|}
\hline & $\mathrm{a} 1$ & $\mathrm{a} 2$ & $\mathrm{a} 3$ & $\mathrm{~d}$ \\
\hline$X_{1}$ & 0.23 & 0.31 & -0.55 & 8.5 \\
\hline$X_{2}$ & -0.48 & -0.6 & 0.51 & 8.2 \\
\hline$X_{3}$ & -0.61 & -0.77 & 1.2 & 8.5 \\
\hline$X_{4}$ & 0.45 & 1.54 & -1.4 & 11 \\
\hline$X_{5}$ & -0.11 & -0.22 & 0.29 & 6.3 \\
\hline$X_{6}$ & -0.51 & -0.64 & 0.76 & 8.8 \\
\hline$X_{7}$ & 0.15 & 0.13 & -0.25 & 10.1 \\
\hline
\end{tabular}

First, getting the binary relation for each attribute using the following relation;

$x R_{a i} y \leftrightarrow|a i(x)-a i(y)| \prec \varepsilon \quad ; i=1,2,3$

for ai take $\varepsilon=0.5$

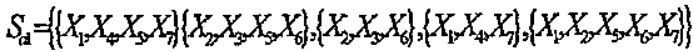

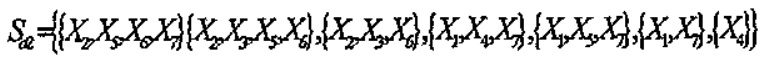
$S_{a}=\left\{\left(X_{2}, X_{3}, X_{3}, X_{6}\right\},\left\{X_{2}, X_{6}\right\},\left\{X_{3}, X_{6}\right\},\left\{X_{5}, X_{6}\right),\left\{X_{1}, X_{7}\right),\left\{X_{4}\right\}\right)$

Then we find the minimal base for each relation as following;
$B m_{e 1}=\left\{\left\{X_{1}, X_{4}, X_{7}\right\},\left\{X_{2}, X_{3}, X_{6}\right\},\left\{X_{2}, X_{6}\right\},\left\{X_{1}, X_{7}\right\},\left\{X_{5}\right\}\right\}$ $B m_{a 2}=\left\{\left\{X_{2}, X_{3}, X_{6}\right\},\left\{X_{1}, X_{7}\right\},\left\{X_{2}, X_{6}\right\},\left\{X_{4}\right\},\left\{X_{5}\right\},\left\{X_{7}\right\}\right\}$ $B m_{03}:=\left\{\left\{X_{2}, X_{6}\right\},\left\{X_{3}, X_{6}\right\},\left\{X_{5}, X_{6}\right\},\left\{X_{1}, X_{7}\right\},\left\{X_{4}\right\},\left\{X_{6}\right\}\right\}$ In order to calculate the reduct and core we shall find;

$$
\begin{aligned}
& B m_{a k a 2 a 3}=\left\{\left\{X_{2}, X_{6}\right\},\left\{X_{3}, X_{6}\right\},\left\{X_{1}, X_{7}\right\},\left\{X_{4}\right\},\left\{X_{5}\right\},\left\{X_{6}\right\},\left\{X_{7}\right\}\right\} \\
& B m_{a \text { la2 }}=\left\{\left\{X_{2}, X_{3}, X_{6}\right\},\left\{X_{2}, X_{6}\right\},\left\{X_{1}, X_{7}\right\},\left\{X_{4}\right\},\left\{X_{5}\right\},\left\{X_{7}\right\}\right\} \\
& B m_{a a_{a 3}}=\left\{\left\{X_{2}, X_{6}\right\},\left\{X_{3}, X_{6}\right\},\left\{X_{1}, X_{7}\right\},\left\{X_{4}\right\},\left\{X_{5}\right\},\left\{X_{6}\right\}\right\} \\
& B m_{a 2 a 3}=\left\{\left\{X_{2}, X_{6}\right\},\left\{X_{3}, X_{6}\right\},\left\{X_{1}, X_{7}\right\},\left\{X_{4}\right\},\left\{X_{5}\right\},\left\{X_{6}\right\},\left\{X_{7}\right\}\right\}
\end{aligned}
$$

Appling the first relation to obtain the binary relation of $\mathrm{d}$ taking $\varepsilon=0.5$

$S_{d}=\left\{\left\{X_{1}, X_{3}, X_{6}\right\},\left\{X_{1}, X_{2}, X_{3}, X_{6}\right\},\left\{X_{1}, X_{2}, X_{3}\right\},\left\{X_{7}\right\},\left\{X_{4}\right\},\left\{X_{5}\right\}\right\}$

Then finding minimal base of decision

$B m_{d}=\left\{\left\{X_{1}, X_{3}, X_{6}\right\},\left\{X_{1}, X_{2}, X_{3}, X_{6}\right\},\left\{X_{1}, X_{2}, X_{3}\right\},\left\{X_{1}, X_{3}\right\},\left\{X_{7}\right\},\left\{X_{4}\right\},\left\{X_{3}\right\}\right\}$

The positive region for each subset of attribute with respect to $d$ are;

$$
\begin{aligned}
& \operatorname{POS}_{B m_{a \mid a z a 3}}\left(B m_{d}\right)=\left\{X_{2}, X_{3}, X_{4}, X_{5}, X_{6}, X_{7}\right\} \\
& \operatorname{POS}_{B m_{a 1 a 2}}\left(B m_{d}\right)=\left\{X_{2}, X_{3}, X_{4}, X_{5}, X_{6}, X_{7}\right\} \\
& \operatorname{POS}_{B m_{a: a 3}}\left(B m_{d}\right)=\left\{X_{2}, X_{3}, X_{4}, X_{5}, X_{6}\right\} \\
& \operatorname{POS}_{B m_{a 2 a 3}}\left(B m_{d}\right)=\left\{X_{2}, X_{3}, X_{4}, X_{5}, X_{6}, X_{7}\right\}
\end{aligned}
$$

From the above; the reduct is $\{\{a 1, a 2\},\{a 2, a 3\}\}$ and the core is $\{a 2\}$.The reduced information system can be viewed as a decision table (2).

Table (2) reduced information system; condition attributes $\{$ a1, a2 $\}$ decision attribute $\{\mathrm{d}\}$.

\begin{tabular}{|c|c|c|c|}
\hline & $\mathrm{a} 1$ & $\mathrm{a} 2$ & $\mathrm{~d}$ \\
\hline$X_{1}$ & 0.23 & 0.31 & 8.5 \\
\hline$X_{2}$ & -0.48 & -0.6 & 8.2 \\
\hline$X_{3}$ & -0.61 & -0.77 & 8.5 \\
\hline$X_{4}$ & 0.45 & 1.54 & 11 \\
\hline$X_{5}$ & -0.11 & -0.22 & 6.3 \\
\hline$X_{6}$ & -0.51 & -0.64 & 8.8 \\
\hline$X_{7}$ & 0.15 & 0.13 & 10.1 \\
\hline
\end{tabular}

Reducts obtained by our method is contained in the reduct obtained using the Discernibility matrix, that means the new approach gives more reduction.

\section{CONCLUSIONS}

Rough sets theory has been applied successfully in many disciplines. One of the major limitations of the traditional rough sets model in the real applications the intersected classes so. In this paper, we have been introduced a new model for rough set theory to solve the problem of intersected classes and new definition 
for lower and upper approximation and a new approach for finding core and reduct attributes.

Our future work will be focusing on the experiments of this model with large data set.

\section{REFERENCES}

[1] Arcewski, T. and Ziarko, W.P. (1988). Adaptive Expert System for Preliminary Design Wind Bracings in Steel Skeleton Structure. Second Century of the Skyscraper, Van Nostrand Reinhold Company, N.Y., pp. 847-855

[2] Bazan, J., Nguyen, S., Synak, P., Worblewski, J.(2000). Rough set algorithms in classification problems, Rough Set Methods and Applications: New Developments in Knowledge Discovery in Information Systems, L. Polkowski, T.Y.Lin, and S. Tsumoto (eds), 49-88, Physica-verlag, Heidelberg, Germany

[3] Hu, X., Cercone N., Han, J., Zirako, W. (2002). GRS: A Generalized Rough Sets Model, in Data Mining, Rough Sets and Granular Computing, T.Y.Lin, Y.Y.Yao and L.Zadeh(eds), PhiscaVerlage, $447-460$

[4] Kumar A.(1998).New Technique for Data Reduction in Database Systems for Knowledge Discovery Applications, Journal of Intelligent Information Systems, 10(1), 31-48

[5] Luba, T., Rybnik, J.(1991). Relation Between Multiple-Valued Logic and Decision Logic with Respect to Rough Set Theory Semantics. Manuscript pp, 1-22
[6] Nguyen, H., Nguyen, S.(1996). Some efficient algorithms for rough set methods, Proc. IPMU Granada, Spain, 1451-1456

[7] Pawlak Z. (1982). Rough sets, international journal of information and computer science, 11(5), 341-356

[8] Pawlak Z. (1992). Rough sets: Theoretical Aspects of Reasoning about data, Kluwer Academic Publishers

[9] Pawlak, Z., Slowinski, K. and Slowinski, R.(1986). Rough Classification of Patients After Highly Selective Vagotomy for Duodenal Ulcer. Int. Journal of Man-Machine Studies, 24, pp. 413-433

[10] Polkowski, L., Skowron, A.(1994). Rough mereology, Proc. ISMIS, Charlotte, NC,85-94

[11] Polkowski, L., Skowron, A.(1996). Rough mereology: A new paradigm for approximate reasoning, J. of approximate Reasoning, 15(4), 333-365

[12] Skowron, A., Stepaniuk, J., Tolerance approximation spaces, Fundmenta Informatica 27(2-3), 245-253

[13] Stepaniuk, J.(1994). Generalized approximation spaces, Pro. Of the $3^{\text {rd }}$ International Workshop on Rough Sets and Soft Computing, San Jose State university, San Jose, California, 156-163

[14] Ziarko, W.(1993). Variable Precision Rough Set Model, Journal of Computer and Science, 46(1), $39-59$ 